\title{
Public Health (PH) Preparedness: Improving Data Exchange for Monitoring PH Threats through Content Standardization
}

\author{
Nikolay Lipskiy* \\ CSELS/DHIS, CDC, Atlanta, GA, USA
}

\section{Objective}

The objective of this presentation is to evaluate progress in developing semantically interoperable content for PH systems that monitor PH threats. Also, it highlights potential solutions for improve standardization of those data exchanges.

\section{Introduction}

Effective PH information exchange depends on standardized data to ensure system-to-system interoperability and is a critical component of preparedness and response. The Common Ground Preparedness Framework (CGPF) was developed through a three-year collaboration of eight state and local health departments to define and categorize $\mathrm{PH}$ business processes related to preparedness to include prepare, manage, monitor, investigate, intervene and recover. The CGPF may be used to prioritize standardization activities.[1] Monitoring, which is the crucial CGPF category for the entire PH preparedness business processes includes assessing population trends, and conducting surveillance.[2]. The author used the CCPF monitoring process as a basis for the comparison to determine those standards that aligned with these processes and identified any gaps in the standards. This assessment may help in better understanding content standardization for preparedness and areas for improvement.

\section{Methods}

The following four CGPF Monitoring business processes were analyzed in our study: Assess Population Trends and Patterns; Conduct Syndromic Surveillance; Conduct Notifiable Disease Surveillance and Conduct Environmental Surveillance [1]. The standardization needs for these processes were assessed against Public Health Information Network (PHIN) standards' repository that contains 62 electronic data exchange standards [3] and 15 data classifications [4]. Based on CDC guidance [5] we made an assumption that the PHIN repositories reflect the landscape of existing $\mathrm{PH}$ standards implemented by CDC and partners' $\mathrm{PH}$ systems. We assessed data elements, value sets and classifications that are included in standards against already defined content categories such as, patient information, encounter, health problems etc. [6] and content categories that support CGPF business processes.

\section{Results}

We found that the methodology used to assess the standards by specific business process and content categories was helpful for purposes of this study. It demonstrates that PHIN content standards supports standardization of all four business processes that comprise the CGPG PH threats Monitoring category. However, we observed differences in the level of content standardization of these processes as well as level of standardization for exchanging of data on patientand population-levels. Specifically, Assess Population Trends and Patterns business process appeared to be on the lowest level of standardization. Existing content standards for the CGPF Monitoring category are primarily oriented for exchange of patient-level data. We found that the PHIN classifications effectively cover contents standardization needs for the CGPF PH threats Monitoring category. However, a level of a codification appeared to be different by content category. For example, existing standards do not provide guidance on mapping of ICD-9/10 codes to syndromes for conducting syndromic surveillance.

\section{Conclusions}

While progress towards content standardization for exchange of data for monitoring of $\mathrm{PH}$ threats exists, there are some gaps that become evident in this study approach. This study demonstrates that content standards for Monitoring PH threats at a patient-level are better defined than at population level.

Results of this study underline the importance of better coordination of data harmonization efforts between and within domains of $\mathrm{PH}$ knowledge.

\section{Keywords}

. Monitoring PH threats; Standardization of surveillance; Content standards

\section{References}

1. Gibson PJ, Theadore F, Jellison JB. The common ground preparedness framework: acomprehensive description of public health emergency preparedness. Am J Public Health, 2012; 102(4):633-642. http://www. ncbi.nlm.nih.gov/pmc/articles/PMC3489381/

2. ISDS. Electronic Syndromic Surveillance Using Hospital Inpatient and Ambulatory Clinical Care Electronic Health Record Data. http://www. syndromic.org/storage/ISDS_2012-MUse-Recommendations.pdf

3. PHIN. Data Interchange Standards. http://www.cdc.gov/phin/resources/ standards/data_interchange.html Assessed on 07/16/2014

4. PHIN. Classifications. http://www.cdc.gov/phin/resources/standards/ classifications.html

CDC. Public Health Preparedness Capabilities: National Standards for State and Local Planning, 2011. http://www.cdc.gov/phpr/capabilities/

5. HHS. HHS/ONC. Standards and Interoperability Framework. Data Harmonization Profile. Version 2.0 http://wiki.siframework.org/ file/view/DataHarmonizationProfile_02132018.docx/428760522/ DataHarmonizationProfile_02132018.docx

\section{*Nikolay Lipskiy}

E-mail: dgz1@cdc.gov 